\title{
Dialoguer avec le discours d'autrui dans les écrits universitaires ou comment construire un statut d'auteur
}

\author{
Sibely Oliveira Silva ${ }^{1}$ \\ Programa de pós-graduação em Letras, Pontifícia Universidade Católica de Minas Gerais, \\ Belo Horizonte, MG, Brasil \\ Françoise Boch ${ }^{2}$ \\ Laboratoire LIDILEM de Sciences du Langage, Université Grenoble Alpes, Grenoble, France
}

Resumé : Si la question du plagiat et, plus globalement, des normes à respecter dans les écrits académiques semble bien représentée dans les discours circulant à l'université, et notamment dans les manuels d'aide à la rédaction destinés aux étudiants, la question fondamentale de la construction d'une posture auctoriale dans l'écriture reste peu abordée, sans doute en raison de sa complexité souvent pointée dans les recherches en littéracies académiques. Comment construit-on son statut d'auteur lorsqu'on est étudiant ? Cette question est ici traitée sous l'angle des procédés utilisés par les étudiants dans le dialogue qu'ils instaurent avec les discours d'autres auteurs. A partir de l'analyse fine de textes académiques produits par des étudiants de niveaux divers du cours de Lettres, cet article identifie les différents procédés linguistiques, discursifs, textuels et énonciatifs mis en œuvre par ces étudiants et discute de leur effets sur le plan de la construction de leur posture d'auteur.

Mots clés : Auctorialité ; Discours d’autrui ; Écriture académique.

Título: Dialogar com o discurso de outrem na escrita acadêmica ou como construir uma posição de autor

Resumo3: Se a questão do plágio e, mais globalmente, das normas a respeitar nos escritos

\footnotetext{
${ }^{1}$ Doctorante en Langue Portugaise et Linguistique (PUC Minas). https://orcid.org/0000-0002-5782$\underline{0101}$

E-mail: sibelyolliver@gmail.com

2 Enseignante-chercheure en Sciences du Langage (Université Grenoble Alpes). https://sites.google.com/site/cvfrancoiseboch/

E-mail: francoise.boch@univ-grenoble-alpes.fr

${ }^{3}$ Ce travail a été réalisé avec le soutien de la Coordination pour l'amélioration du personnel de l'enseignement supérieur - Brésil (CAPES) - Code de financement 001 et est lié au Projet CAPESCOFECUB (Edital 19/2014 no 834-15). La présente contribution est la version remaniée d'une communication intitulée "Dialoguer avec le discours d'autrui dans l'écrit académique: analyse de pratiques estudiantines" et présentée par les deux auteures à la Journée d'étude "Letramentos/Literacies/Littéracies : Regards croisés sur des problématiques de recherche internationales (Projet CAPES-COFECUB - Edital 19/2014, no 834-15), le 13/12/2018 à l'Université de Lille-France.
} 
acadêmicos parece bem representada nos discursos que circulam na universidade, notadamente nos manuais de ajuda para redação destinados aos estudantes, a questão fundamental da construção de uma posição autoral na escrita resta ainda pouco abordada, sem dúvida em razão de sua complexidade frequentemente pontuada nas pesquisas sobre os letramentos acadêmicos. Como construir o estatuto de autor quando se é estudante? Essa questão é aqui tratada sob o ângulo dos procedimentos utilizados por estudantes no diálogo que instauram com o discurso de outros autores. A partir da análise fina de textos acadêmicos produzidos por estudantes de diferentes níveis do curso de Letras, este artigo identifica os diferentes procedimentos linguístico-textuais, discursivos e enunciativos utilizados por esses estudantes e discute seus efeitos sobre o plano da construção de sua posição autoral.

Palavras-chave: Autoria; Discurso de outrem; Escrita acadêmica.

Title: In Dialogue with the speech of others in academic writing or how to construct an author's position

Abstract: The issue of plagiarism in academic writing has been widely treated and much research also exists on general academic writing norms. This is most evident in student writing manuals. However, one fundamental question is much less frequently addressed: how do students construct their authorial stance? This is perhaps due to the complexity of the process, which has been frequently mentioned in academic literacy research. This article seeks to answer this question by analyzing the dialogue student writers establish when referring to other authors. Academic texts written by students at different levels of study (in the field of Literature) were collected and analyzed to show how students mobilize different linguistic, discursive, textual and enunciative processes. This provides new insight into how student writers construct their authorial posture.

Keywords: Authorial stance; In-text citations; Academic writing.

\section{Le dialogue avec la parole d'autrui et sa relation avec l'auctorialité dans l'écriture académique}

Sujet constamment débattu au sein de l'université, l'auctorialité se révèle une notion centrale dans le champ de la littéracie universitaire. Cette notion est traditionnellement envisagée à travers le filtre du plagiat, probablement en raison de la forte présence dans les bibliothèques universitaires des manuels méthodologiques d'aide à la rédaction de textes académiques. On y trouve majoritairement la défense d'une conception normative/prescriptive et parfois standardisée des pratiques d'écriture académique. II n'est pas rare que cette vision normative de l'écriture soit également partagée par les enseignants, et ce dans différentes disciplines.

Tout en reconnaissant, dans une certaine mesure, la contribution de ces manuels ou des travaux empruntant la même perspective à la formation des étudiants à l'écriture académique, il nous semble toutefois indispensable de considérer, comme le souligne notamment Grossmann (2015), l'existence d'une variété de pratiques scientifiques qui sont inévitablement guidées par des pratiques d'écriture différentes. 
En ce sens, nous souhaitons remettre en question certaines consignes données aux étudiants, souvent lapidaires et peu discutées circulant autour de l'écriture académique (telles que " évitez de faire de longues citations ", " évitez d'utiliser citation de citation » ou " "évitez de remplir le texte de citations", pour en citer quelques-unes) et nous concentrer sur le thème du dialogue avec le discours d'autrui et son rapport avec la construction de la position d'auteur. Le dialogue avec la parole d'autrui abrite à nos yeux d'autres valeurs qu'une vision normative, qui doivent être considérées en formation. S'approprier l'écriture académique passe par exemple par la prise de conscience fondamentale que que l'image/le statut d'un auteur est avant tout lié à la façon dont il se construit discursivement et énonciativement dans le dialogue avec d'autres discours.

Ainsi, loin de cette vision conventionnelle, parfois techniciste ( comment citer sans plagier »), voire moraliste (" plagier est un délit ») de l'insertion du discours d'autrui dans son texte, l'approche discursive du rapport au discours d'autrui par l'auctorialité nous semble plus riche et plus productive en formation, même si plus complexe à saisir et à didactiser.

Comme le souligne Compagnon (2007), il est impossible de traiter la question de la citation sans l'associer à l'auteur: en même temps qu'elle travaille le texte, la citation travaille aussi l'auteur, " parce qu' elle ne se produit que dans un travail qui la déplace et le fait agir" (COMPAGNON, 2007, p.47). En ce sens, l'auteur doit adopter une attitude responsable et responsive avec et à partir d'elle, au sens bakhtinien.

En effet, dans son processus d'appropriation de l'écriture, dont résulte la légitimation de sa propre identité académique, l'étudiant-auteur donne à voir de lui-même dans les discours avec lesquels il dialogue, à la fois à travers l'univers social et discursif de son domaine disciplinaire et les sources auxquelles il recourt.

Cette position est proche de la vision de Grossmann (2003), pour qui les pratiques de citation renvoient inévitablement à la question de l'auteur, non seulement parce que citer l'autre est un acte codifié, qui répond à des normes permettant d'éviter le plagiat, mais surtout parce que l'auteur citant choisit les auteurs qui l'aident à établir son propre dire.

En ce sens, la citation peut être envisagée dans le cadre plus large de la polyphonie, dont la gestion doit être intégrée au sein des compétences à développer pour entrer dans l'écriture académique (BOCH; GROSSMANN, 2001).

Dans ce jeu dialogique constitutif de l'écriture, l'appropriation du discours d'autrui et la nécessité de dialoguer dans son texte avec ces discours représentent un défi majeur pour l'étudiant dans son initiation à l'écriture académique. "Dialoguer " suppose en effet un ensemble d'actions complexes : prise de position, choix des sources, évaluation sur le dire et sur le dit, relation entre concepts et idées, jeu d'images, etc.

Nous faisons ici l'hypothèse que la position auctoriale est indexée à toutes ces actions qui, dans un réseau d'interdépendance, concourent à la construction de l'image d'un auteur. 
Nous nous inscrivons ainsi dans une approche dialogique bakhtinienne de la langue et du discours, et empruntons également à Bakhtine la notion d'auctorialité (1997).

Assumer la notion d'auctorialité dans une perspective bakhtinienne impose de considérer le principe exotopique de la création esthétique, selon lequel l'auteur-créateur doit assumer différents lieux ou déplacements qui le conduisent à percevoir l'ensemble de l'œuvre en adoptant un regard distancié et englobant (" un surplus de vision " chez BAKHTINE, 1997). Selon Bakhtine, ce regard est toujours modulé par la relation entre l'auteur et l'autre et par la nécessité du retour de l'auteur à sa position singulière pour, après la rencontre avec l'horizon de l'autre, agir sur l'objet esthétique en le formant selon sa position axiologique. Dans cette perspective, tout chercheur-auteur ( $y$ compris les étudiants que nous devons former à cette tâche complexe) se positionne, dans l'écriture académique, en adoptant un regard critique sur les voix avec lesquelles ils dialoguent : ils sont capables, en termes bakhtiniens, de rendre compte de l'ensemble de ces voix, en leur attribuant une voix singulière, imprégnée d'une tonalité expressive orientée par de nouveaux faisceaux de valeurs axiologiques, c'est-à-dire de nouveaux sens, idées et (re) positionnements.

À ce propos, Possenti (2009, p.113), au cours d'un débat sur les indices de l'auctorialité, affirme que " quelqu'un devient auteur lorsqu'il adopte fondamentalement deux attitudes : il donne la parole aux autres et reste à l'écart du texte ", c'est-à-dire qu'il est capable d'exercer un mouvement exotopique pour évaluer (de l'extérieur) le discours d'autrui par rapport à sa propre voix. Ainsi, nous comprenons que l'auctorialité est construite dans une relation dialogique qui autorise la (ré)appropriation des discours par le sujet-auteur. Toujours en appui à Bakhtine, nous croyons que cette appropriation est observable à travers l'intention discursive du sujet-auteur, mais aussi ce qu'il dénomme "sa tonalité expressive ", son positionnement énonciatif, son style individuel, dans les diverses situations d'interaction auxquelles il est confronté dans l'écriture académique. Nous choisissons ainsi certains de ces lieux d'observations pour étudier les textes d'étudiants, catégories d'analyse que nous détaillerons infra (cf. section 2) et dont nous cherchons ici à observer les manifestations.

Via ces descriptions fines des pratiques estudiantines, notre objectif est de nourrir la recherche en littéracies académiques, partant du principe défendu notamment par Rinck, Boch et Assis (2015, p. 18), que "les recherches sur les littéracies académiques doivent permettre de donner une base théorique solide aux formations pour l'écriture proposées à l'université".

\section{Contexte de la recherche : les ateliers d'écriture académique}

La recherche $e^{4}$ prend appui sur des ateliers réalisés avec un groupe d'étudiants en

\footnotetext{
${ }^{4}$ Recherche-action menée par Sibely $\mathrm{O}$. Silva, issue d'un travail de doctorat encore en cours, réalisé dans le cadre du Programme de Doctorat en Langue Portugaise et Linguistique d'une université
} 
formation initiale de différentes périodes ${ }^{5}$ du cursus de Lettres dans une université privée de Belo Horizonte (Brésil).

Cette formation, intitulée Ateliers de production de textes académiques: démystifier et affronter l'écrit dans l'université, est composée d'un ensemble de neuf ateliers, réalisés au cours de douze séances ${ }^{6}$ avec les étudiants participants, entre mars et juin 2018.

Comme l'indique son titre, le programme de cette formation a été pensé en tenant compte d'un ensemble de représentations et/ou de mythes entourant l'écriture à l'université, comme déjà mencioné, portant notamment, outre la question de la citation déjà évoquée, sur la question de l'objectivité et la neutralité supposées de l'écrit académique. Reuter (1998) l'a bien montré : les représentations précédemment soulignées peuvent faire obstacle au processus d'appropriation de l'écriture académique des étudiants en formation initiale, et notre première tâche en formation consiste à amener les étudiants à en prendre conscience. Plus précisément, la formation avait pour objectif de revisiter la perspective normative (qui est souvent aussi celle des étudiants) du dialogue avec le discours d'autrui et de familiariser les étudiants à une approche plus discursive de cette composante de l'écrit académique.

Ainsi, concernant l'utilisation de la citation, les représentations que se forgeaient les étudiants de l'écrit scientifique ont été interrogées au cours des ateliers, donnant lieu à des discussions autour de questions ${ }^{7}$ telles que : qu'est ce qui est en jeu quand on choisit de faire une citation ?; Que peut dire la citation de celui qui cite ?; Quelles sont les relations de pouvoir qui se jouent dans l'acte de citer ? Une autre question traitée dans la formation, en lien direct avec notre problématique, a largement était évoquée : comment la citation peutelle servir de pont à l'inscription du sujet dans le discours, concernant la construction d'une voix auctoriale?

Parallèlement, la question de l'auctorialité a été traité centralement dans les ateliers, à travers la citation comme objet d'enseignement et d'apprentissage, toujours discuté dans une perspective discursive-dialogique.

Les ateliers sont conçus en deux étapes : une première étape au cours de laquelleles

brésilienne (PUC-Minas). La thèse de doctorat porte sur les effets des pratiques de réécriture dans le développement de la position auctoriale d'étudiants brésiliens en formation initiale inscrits en Lettres. La recherche a été soumise au Comité d'Éthique de la PUC Minas et a obtenu un avis favorable.

${ }^{5} \mathrm{Au}$ Brésil, la période correspond à un temps de formation universitaire équivalent à un semestre.

${ }^{6}$ D'une durée d'1h20, ces réunions ont été réalisées une fois par semaine avec la participation de 18 étudiants. Les ateliers ne faisaient pas partie du programme de l'université et ont été conçus spécifiquement dans le cadre de la recherche-action. Diffusé dans l'institution (affiches présentes dans les salles de cours et sur les réseaux sociaux), le programme a suscité l'inscription d'une vingtaine d'étudiants.

${ }^{7}$ Nous nous situons ici dans la lignée de Paula $(2017$, p.88) qui traite également de telles questions. 
étudiants examinent et discutent de petits textes-sources qui traitent de l'écriture académique, et plus précisément de la notion d'auctorialité. Ces textes peuvent également représenter pour les étudiants des exemples à explorer pour construire leur réflexion sur la façon dont le dialogue avec la parole d'autrui s'y établit. La deuxième étape est consacrée à la production textuelle, toujours articulée à ces discussions. Dans ces ateliers, la priorité est pleinement accordée à la didactique du discours académique et scientifique : l'objectif visé est de permettre aux étudiants de s'approprier des manières de dire spécifiques aux différents genres académiques travaillés, sans toutefois s'apparenter à une approche méthodologique de ces genres.

Notons enfin que dans ces ateliers, les participants sont systématiquement confrontés à différentes pratiques d'écriture et de réécriture fédérées autour du discours d'autrui, dans une perspective discursive.

\section{Démarche méthodologique}

Avant d'entrer dans les détails de la méthodologie adoptée et afin que le lecteur puisse comprendre le cadre dans lequel ont été réalisées les productions analysées dans la section 4, nous fournissons ici quelques précisions sur la nature du $7 \mathrm{e}^{8}$ atelier.

II est nécessaire de préciser que dans les séances précédants le $7 \mathrm{e}$ atelier, le thème du dialogue avec le discours d'autrui et de son rapport avec la construction d'une position d'auteur dans l'écriture académique a fait l'objet d'échanges nourris au sein du groupe. Ces échanges ont émergé des activités pédagogiques réalisées sur la citation, qui exigeaient une posture critique et réflexive des étudiants sur la citation et sur sa relation avec l'auctorialité. Nous nous situons ainsi dans la perspective didactique défendue actuellement dans le champ des littéracies avancées, consistant à former à et par l'écriture (cf. par exemple RINCK, 2011).

Considérant ce parcours, avec le 7e atelier, les étudiants ont eu la possibilité de conjuguer les savoirs acquis pour mener à bien le dialogue avec d'autres voix dans la production de leurs textes, car le but de cet atelier était d'une part de stimuler l'action de textualisation impliquée dans les modes de dire du genre recension académique thématique, d'autre part de favoriser une posture critique, de réflexion et d'analyse.

\footnotetext{
8 II convient de clarifier qu'avant la 7e séance, II a été travaillé avec les étudiants de le 6e séance deux recensions produites par différents auteurs (Rodolfo llari et Helem Cristiane Aquino dos Anjos Fernandes) traitant du même sujet - "Pourquoi (ne pas) enseigner la grammaire à l'école ?", dans le but de discuter avec les étudiants de la manière dont les auteurs des deux textes se sont construits discursivement et énonciativement dans les critiques analysées, ainsi que de promouvoir une réflexion sur les manières de dire intrinsèques à ce genre.
} 
Les étudiants devaient produire un écrit relevant de ce genre dont la thématique était la suivante : "Le travail du sujet sur et dans la langue/le langage et la nécessité de construire une posture auctoriale dans l'écriture académique ».

Selon Assis (2014, p. 543-561) " la recension académique thématique se développe autour d'un thème et, pour cela, il dialogue avec au moins deux textes- sources ". II s'agit donc d'un genre qui requiert de la part de l'étudiant une posture critique et la mise en œuvre de sa capacité de réflexion et d'analyse. Par ailleurs, ce genre étant essentiellement polyphonique, son élaboration demande aux étudiants de bien gérer les différentes voix en présence. Cette gestion des voix est non seulement indispensable à la construction d'une voix auctoriale, mais, plus généralement, elle l'est aussi dans la production de tout texte académique : dans les routines d'écriture universitaire, des étudiants sont confrontés à interagir avec différents discours de théoriciens et à conjuguer des savoirs, des idées, des concepts à travers le dialogue avec d'autres voix.

Cette activité de production reposait sur quatre textes-sources qui traitaient des notions de dialogisme, d'auctorialité et d'écriture. Soulignons ici que la thématique de ces textes comportait une dimension méta-discursive, et la consigne une forme de mise en abyme : les étudiants ont été confrontés à des textes traitant de notions spécifiques dialogisme et auctorialité dans l'écriture - qu'ils devaient, par ailleurs, mettre en œuvre à travers leur écriture, et par là, témoigner d'une certaine compétence à l'égard de ces notions.

\section{Recueil et traitement des données}

Le corpus traité dans cet article est composé de 35 copies/textes produits dans le cadre des $3 e^{9}$ et $7 e$ ateliers. Ce corpus est analysé dans une perspective discursive : l'analyse vise à identifier les stratégies de nature linguistiques-textuelles, discursives et énonciatives mobilisées par les étudiants participant à la recherche, lorsqu'ils dialoguent avec d'autres auteurs (ou font dialoguer les auteurs entre eux), stratégies qui peuvent révéler la construction de la position d'auteur de ces étudiants.

Pour la sélection du corpus principal d'analyse (extraits de textes), nous adoptons les critères de choix suivants :

(i) textes produits par des étudiants de différentes périodes (deux étudiants de la 2ème période et un étudiant de la 9ème période du cours de Lettres), afin de présenter un portrait représentatif de la classe et de ses performances par

\footnotetext{
${ }^{9}$ Dans la 3e séance, les étudiants devaient notamment produire un texte reliant les idées centrales des textes discutés, visant à construire un point de vue (en termes d'accord ou de désaccord) vis à vis des discours avec lesquels ils dialoguaient.
} 
rapport à l’activité proposée;

(ii) extraits des recensions produites par les étudiants dans le contexte du 7eatelier, afin de favoriser le développement d'une position d'auteur, sous-tendu par une posture critique, de réflexion et d'analyse à travers le dialogue avec le discours d'autrui ;

(iii) extraits qui illustrent une position d'auteur réussie par rapport aux trois catégories analysées, et un extrait représentant un positionnement d'auteur moins réussi.

Trois catégories d'analyse, empruntées à Bakhtine, ont été retenues : la tonalité expressive, le positionnement discursif et le travail du sujet sur et dans la langue/le langage sont considérés comme constitutifs de la notion d'auctorialité adoptée dans le cadre bakhtinien (cf. section 1). Bien que ces catégories d'analyse soient en réalité imbriquées, nous choisissons de les discuter ici séparément, à seule fin de clarification méthodologique. Pour bien illustrer dans cette contribution le travail mené auprès des étudiants, nous avons sélectionné quatre extraits de textes, tous produits dans le contexte du 7 e atelier.

Un tableau récapitule, à la fin de la section 4, les stratégies relevant de ces trois catégories et observables dans l'ensemble du corpus de 35 textes produits par les étudiants participant aux $3 e$ et $7 e$ ateliers.

Précisons enfin que dans la partie qui suit, nous utilisons les termes de " discours cité " et " discours citant ". "Discours cité " renvoie ici à tout discours d'autrui, que ce discours soit sous la forme d'une citation ou d'une reformulation. "Discours citant " renvoie au discours tenu par l'étudiant-auteur lorsqu'il fait référence au discours d'autrui.

\section{Le regard sur les données : la construction de l'auctorialité}

Nous cherchons donc à analyser la manière dont les étudiants participant aux ateliers dialoguent avec le discours d'autrui, notre hypothèse étant que la dimension dialogique, impliquant elle-même "l'être et le dire avec et à partir d'autrui " (Assis, 2014), donne des indications sur la posture auctoriale que se construit l'auteur-étudiant dans son texte. L'analyse présente dans un premier temps trois extraits qui révèlent la manifestation d'une posture auctoriale réussie. 
Illustrations ${ }^{10}$ de postures auctoriales réussies dans le dialogue avec le discours d'autrui

Le premier exemple présente le point de vue d'un étudiant-auteur sur le processus d'écriture.

\section{Exemple 1 La tonalité expressive}

L'acte d'écrire comporte en soi plusieurs étapes : c'est un «jeu» énonciatif dans lequel le joueur doit réfléchir à quelle stratégie il va utiliser, quels effets elle va causer, et quelles voix seront " invitées » à composer la production textuelle. Il est demandé d'écrire un texte qui présente un travail réflexif et minutieux basé sur un modus operandi qui, dans une certaine mesure, se passe de cette manière :

J'ai écrit ce texte que vous allez lire maintenant. Mais je ne l'ai pas écrit en un seul jet, dans un seul souffle, n'utilisant les mots que pour exprimer une pensée déjà prête, fruit d'une inspiration transcendante. Non. Je tissais des sens quand j'écrivais, j'inventais, je me rappelais, je m'appropriais, je créais, je reformulais, je précisais, j'effaçais, j'abandonnais, je choisissais, j'élisais des sens, je faisais taire les autres. C'est-à-dire que j'enlaçais des mots, j'intercalais des contextes, j'entrelaçais des textures, tisserande que je suis aussi. (ARAÚJO, 2001).

Source: extrait de la 3ème version de la recension (partie développement) d'un étudiant de la 9ème période.

Avant d'appréhender ce texte, rappelons notre première catégorie d'analyse : la tonalité expressive. Dans la perspective bakhtinienne, la tonalité expressive est définie comme " un des moyens d'exprimer le rapport émotif-valoriel du locuteur à l'objet de son discours ». (BAKHTINE, 1997, p.310).

Elle peut se révéler à travers la présence de la subjectivité de l'étudiant-auteur, dans la coloration lexicale des mots qu'il utilise, les nuances qu'il apporte, les figures de style.

Dans l'exemple 1, l'auteur a recours à la métaphore (« joueur » ou « jeu d'énonciation ») pour présenter sa définition du processus qu'implique l'écriture. L'emploi de la métaphore, à notre avis, témoigne d'un mouvement argumentatif sophistiqué et révèle une

\footnotetext{
${ }^{10}$ Dans tous les extraits présentés dans cette section, ont été préservées la forme originale des textes produits par les étudiants ainsi que leur manière de faire référence au discours d'autrui.
} 
compréhension active du discours d'autrui : un tel choix se montre cohérent avec la perspective énonciative du discours cité et (ré) actualise le sens donné à ce discours en même temps.

Dans cette relation dialogique, le discours cité a une double expression : la sienne et celle de l'énoncé qui l'accueille (BAKHTINE, 1997).

Ainsi, de notre point de vue, l'étudiant-auteur se montre capable de comprendre la conception de l'écriture qui balise la citation qu'il choisit. Dans cette mesure, l'utilisation de la métaphore du " jeu ", associée aux mots " stratégies " et " étapes ", corrobore la conception de l'écriture en tant que processus, comme on peut le vérifier dans une telle citation. Ce choix judicieux de la métaphore manifeste de la part de l'étudiant-auteur une réelle appropriation du discours cité, avec lequel il entre en dialogue.

Il s'agit donc d'une stratégie discursive visant à clarifier le point de vue de l'étudiantauteur ("L'acte d'écriture lui-même comporte plusieurs étapes ; Le joueur doit réfléchir à quelle stratégie il va utiliser, quels effets elle va causer, et quelles voix seront "invitées " à composer un texte»), puisque comme on le sait, la métaphore est généralement utilisée pour faire comprendre. Elle donne au discours académique un ton didactique (étant plus imagé, le discours est a priori plus accessible). En même temps, l'emploi de la métaphore place l'étudiant-auteur dans une position dominante en ce qu'il articule étroitement son discours avec celui de l'auteur. En d'autres termes, le recours à la métaphore instaure l'image d'un discours de crédibilité à travers lequel l'étudiant-auteur vise l'adhésion de l'interlocuteur.

\section{Exemple 2 Le positionnement discursif dans le dialogue avec le discours d'autrui}

Extrait du même ${ }^{11}$ texte que l'exemple 1, cet autre exemple témoigne d'un positionnement fort de l'étudiant-auteur par rapport au sujet traité (le travail du sujet du discours sur la langue / le langage et la construction de son image d'auteur dans l'écriture). Cet extrait correspond à l'introduction de la recension.

Le travail du sujet du discours sur la langue / le langage peut se réaliser de plusieurs manières ; la façon dont ce travail est effectué définit comment ce sujet se constitue en tant qu'auteur. Son identité d'auteur est composée à partir des types de discours qu'il réfute ou endosse. Les stratégies textuelles et linguistiques-discursives utilisées sont des éléments qui affectent directement la constitution de son identité auctoriale. Dans le travail textuel de l'auteur, nous pouvons mettre en évidence le dialogue avec le discours d'autrui, ce qui nous

\footnotetext{
${ }^{11}$ Nous précisons que nous utilisons un autre extrait du même texte, parce que l'extrait retenu est particulièrement réussi et il illustre bien ce qu'on attend. Les autres autres extraits sont en cours de traitement et l'analyse globale sera présentée dans la thèse.
} 
renvoie au fait qu'il est impossible de " décoller » notre discours de ceux/celui de tiers, une fois que

la parole fait toujours allusion, même contre sa volonté, qu'on le sache ou non, à la parole de l'autre. II n'y a pas de parole jugement, de parole sur objet, qui ne soit pas une parole-allocution, une parole qui entre dialogiquement au contact avec une autre parole, parole sur parole et visant à la parole. (PONZIO, 2010).

Il est donc plausible de remettre en question l'originalité absolue de tout texte ou discours, puisque chaque mot et toute la production textuelle dialogue, dans une certaine mesure, ou reprend un certain énoncé de quelqu'un. Ainsi, on peut affirmer que tout texte se configure comme un intertexte d'un immense "réseau énonciatif » dont il est un élément constituant. On peut citer, comme exemple de ce dialogue interdiscursif, le genre de la critique qui apporte différents modes de dire,

surtout en ce qui concerne les formes d'appropriation du discours d'autrui et la construction d'une position énonciative à travers laquelle l'écrivain dessine sa compréhension, son point de vue sur l'objet du dire et ses relations avec les voix convoquées et le champ scientifique en question. (ASSIS, 2014).

Source: extrait de l'introduction de la 3ème version de la recension d'un étudiant de la 9ème période.

On note ici que l'étudiant-auteur inscrit d'emblée le discours d'autrui dans une orientation argumentative, en faisant pénétrer le discours cité dans le discours citant pour mettre en relief et soutenir les idées auxquelles l'étudiant-auteur adhère. Cette intention est visible, par exemple, dans la manière dont la première citation est introduite : la structure syntaxique adoptée induit une continuité de pensée entre les deux discours (" une fois que le mot fait toujours allusions à [...] »).

Un tel choix crée un effet d'unité dans les voix du discours sur le plan énonciatif, mettant ainsi en évidence le positionnement du discours citant : " Dans le travail textuel de l'auteur, nous pouvons mettre en évidence le dialogue avec le discours d'autrui, ce qui nous renvoie au fait qu'il est impossible de 'décoller' notre discours de ceux/celui de tiers, une fois que $[. .$.$] , et non le contraire ».$

Dans le dialogue entre discours citant et discours cité, le premier assume une position de chef d'orchestre des voix, en montrant ainsi l'effort de l'étudiant-auteur pour construire 
un éthos scientifique de l'autorité. Cet éthos se matérialise dans le choix pertinent des citations, dans le souci de persuasion des pairs avec qui on dialogue dans la communauté discursive, à travers d'une part le recours à l'exemple (On peut citer comme exemple) pour élucider le dit, et d’autre part le ton épistémique du discours, révélé dans les explications.

À ce sujet, il convient de noter que le premier paragraphe est entièrement constitué d'assertions, suivi d'explications : "Le travail du sujet du discours sur la langue /le langage peut se réaliser de plusieurs manières ; la façon dont ce travail est effectué définit comment ce sujet se constitue en tant qu'auteur ». En outre, l'étudiant-auteur établit des relations entre les concepts (" réseau énonciatif ", " intertexte ", " interdiscours ") en proposant des idées nouvelles par rapport au discours avec lequel il dialogue. Autrement dit, sa manière à lui d'articuler très finement discours citant et discours cité est en soi un témoignage (voire une preuve) du point de vue contenu dans ces discours.

Dans l'extrait présenté, on peut observer la prise de position de l'étudiant-auteur par rapport aux voix convoquées, ainsi que par rapport au propre cadre théorique discuté, action qui se réalise, par exemple, par l'établissement de relations entre les concepts déjà mentionnés.

Il est intéressant de noter que l'établissement de ces relations se réalise par la manifestation d'un positionnement de l'étudiant-auteur par rapport à l'objet de discussion, ici sur la conception de l'écriture dialogique. Il établit de telles relations afin d'expliquer le point de vue défendu, qui est précisément une problématisation de l'idée de l'auctorialité liée à la conception de propriété intellectuelle (" [...] I/ est donc plausible de remettre en question l'originalité absolue de tout texte ou discours [...]»).

Du point de vue persuasif de l'usage des citations, les choix effectués par l'étudiantauteur nous semblent pertinents : les citation explicitent de manière cohérente le point de vue de l'étudiant et lui confèrent une plus grande force locutoire. Ainsi, l'étudiant-auteur utilise à bon escient $t$ le discours d'autrui pour servir son orientation discursive.

Dans le premier cas, le discours cité est marqué par des expressions très tranchées telles que : " le mot se réfère toujours, même contre sa volonté, à la parole de l'autre [...] » (Avec " toujours ", Ponzio accrédite l'affirmation de l'étudiant-auteur selon laquelle il est impossible d'envisager un discours non dialogique). De ce fait, la citation est balisée par un discours de certitude, dont témoigne nettement le nombre élevé d'assertions (" II n'existe pas de parole jugement, de parole sur objet, qui ne soit pas une parole-allocution [...] "). Cette orientation discursive nous semble renforcer et assurer le positionnement énonciatif de l'étudiant-auteur.

Dans le cas de la deuxième citation, l'étudiant-auteur utilise la stratégie discursive consistant à mentionner le genre recension ( "On peut citer, comme exemple de ce dialogue interdiscursif, le genre recension qui apporte différents modes de dire ", et comment il se 
configure dans la relation entre plusieurs voix, pour valider et illustrer l'idée que l'écriture se construit dans et par l'interdiscours: "surtout en ce qui concerne les formes d'appropriation du discours d'autrui et la construction d'une position énonciative à travers laquelle l'écrivain dessine sa compréhension, son point de vue sur l'objet du dire et ses relations avec les voix convoquées et le champ scientifique en question. (ASSIS, 2014)». il s'agit d'un auteur qui s'engage dans le discours en adoptant une position argumentative.

Dans ce contexte, il convient de rappeler la position de Boch (1998), qui se référant à Coltier (1988, p.27), souligne que "l'exemple permet au scripteur d'assurer la bonne réception de son discours par le destinataire; l'exemplification est donc une trace de l'effort consenti par le scripteur pour que son discours soit reçu comme il entend qu'il le soit ».

Le recours à l'exemple serait donc un moyen de "répondre ", dans le sens bakhtinien du terme, à une possible " contre-parole " d'un interlocuteur potentiel. En d'autres termes, l'étudiant-auteur démontre avec une telle stratégie avoir bien identifié d'une part le jeu établi entre les pairs dans la communauté discursive et d'autre part les propres injonctions du discours académique-scientifique (toute affirmation nécessite explication et justification).

En outre, compte tenu de la teneur discursive des citations sélectionnées par l'étudiant-auteur, on peut penser qu'il a fait sciemment le choix de citations littérales et non de reformulations. Le choix de ces citations peut être interprété comme un indice de la compétence de l'étudiant-auteur, sensible aux effets de sens générés par un mode de référence au discours d'autrui ou un autre dans le contexte précis du ponto de vista qu'il veut transmettre à son interlocuteur.

Par conséquent, l'étudiant-auteur présente une compréhension active du discours cité en articulant les idées entre le dire et le dit. Ces indices linguistiques-textuels et discursifs montrent, ainsi, l'émergence d'un positionnement énonciatif en pleine négociation avec le discours d'autrui et son interlocuteur potentiel, comme nous pouvons l'observer dans le commentaire suivant la première citation : "Ainsi, on peut affirmer que tout texte se configure comme un intertexte d'un immense "réseau énonciatif "dont il est un élément constituant. On peut citer comme exemple de ce dialogue interdiscursif, le genre de critique qui apporte différents modes de dire [...] ».

Nous pouvons en conclure que dans les exemples 1 et 2 , en dialoguant avec le discours d'autrui, l'étudiant-auteur se construit discursivement et énonciativement dans un éthos d'autorité et de crédibilité dans le discours académique. De son texte apparait ainsi une voix auctoriale marquée par l'expression de nouvelles idées, un recours à l'exemple, un effort de mettre en perspective les concepts et d'exprimer des nuances relatives au discours d'autrui. L'étudiant ne se contente pas d'une simple mention ou répétition du discours d'autrui : il s'en distancie et y apporte une réelle valeur ajoutée. 


\section{Exemple 3 Le travail du sujet sur et dans la langue/le langage}

Afin de discuter la pertinence de la catégorie " travail du sujet sur et dans la langue/le langage ", on s'appuiera sur un extrait tiré de la troisième version de son texte de recension (étudiant de 2ème période). L'extrait est issu du développement du texte.

Bakhtine, dans "La forme spatiale du personnage" (2011), et Augusto Ponzio, dans le texte "Altérité et dialogicité de la parole" (2010), expliquent la nécessité de voir le monde du point de vue de l'autre : en faisant usage de la parole des autres, il faut maintenir l'unicité (ou l'originalité) de l'autre. C'est précisément cette altérité - la capacité de se placer à la place d'un autre - qui favorisera l'interaction des savoirs et des manières de dire, et avec elles, la possibilité de construction du sujet auctorial, avec son propre répertoire d'idées et son bagage de connaissances.

Conscient de cette double orientation des mots - par rapport au thème du discours et par rapport à l'autre - le sujet qui se veut auteur doit comprendre qu'écrire avec autorité sur ce qu'il dit et comment il le dit, de façon claire et cohérente, implique un effort, une méthode, un travail acharné et méticuleux.

Source: extrait de la 3ème version de la recension d'un étudiant de la 2ème période.

On observe dans cet extrait les actions méta-discursives de l'étudiant-auteur qui selon nous révèlent, dans une certaine mesure, que le discours citant établit une altérité par rapport à lui-même, si l'on se réfère à Authier-Revuz (1990). Ainsi, on observe un travail sur et dans la langue/ le langage / à travers ce jeu d'altérité, qui met en scène un jeu d'images entre le je-auteur et l'autre.

Le travail du sujet sur la langue/le langage dans la construction du texte et du discours s'avère important, dans cette analyse, pour l'identification des stratégies linguistiques qui peuvent également révéler l'inscription du sujet dans le discours. En ce sens, cette catégorie est liée à la notion de style, conçue ici encore dans la perspective bakhtinienne, selon laquelle " le style dépend de la manière dont le locuteur perçoit et comprend son destinataire, et de la manière dont il suppose une compréhension responsive active » (BAKHTINE, 1997, p.324) .

On peut percevoir ces traces de l'inscription du sujet dans le discours, dans l'exemple 3 , en orientant le regard davantage sur comment c'est dit (le dire) que sur ce qu'il dit (le dit) précisément.

Dans ce cadre, il est important de souligner les mouvements méta-énonciatifs mobilisés (matérialisés par l'utilisation de tirets et de parenthèses). Ces mouvements, qui reprennent le dit pour l'expliquer, supposent, à notre sens, une double compétence chez l'étudiant-auteur. D'une part, l'étudiant-auteur a bien conscience de la non-transparence du 
langage et/ou du discours: il s'agit donc d'un travail de soi dans le discours, travail qui a pour objectif d'assurer la compréhension du point de vue par son interlocuteur. D'autre part, on peut penser que l'étudiant-auteur est conscient de la construction de son image d'auteur dans l'écrit académique: ainsi, "l'arrêt sur le mot " (AUTHIER-REVUZ, 2011) altérité, par exemple, peut être interprété comme une action réussie du point de vue argumentatif (tel que le discours académique l'exige), car l'altérité est une notion centrale dans le cadre théorique concerné.

Le même cas de figure se présente un peu plus loin, avec l'expression cette double orientation des mots, formule qui renforce également la relation entre auteur et interlocuteur, démontrant ainsi une certaine « responsivité et par conséquent, un jugement de valeur " (BAKHTINE, 1997, p.333) de la part de l'étudiant quant à son rôle d'auteurdétenteur de connaissances.

\section{Illustration d'une posture auctoriale faiblement révélée}

A l'inverse des exemples précédents, l'analyse des stratégies linguistiques-textuelles, discursives et énonciatives dans l'exemple suivant, révèle des indices d'une position auctoriale faiblement révélée. L'extrait est tiré d'un texte d'un étudiant de 2ème période.

\section{Exemple 4}

[...] En outre, le positionnement d'auteur d'un sujet, lorsqu'il écrit, ne se réalise pas "ainsi, dans un seul jet et d'un seul souffle, en utilisant des mots uniquement pour exprimer une pensée déjà prête" : comme le défend Liane Castro de Araújo dans son texte "Tisser les sens : réécriture et reproduction de le texte "(2011), le mot " n'a pas été créé pour orner, pour briller comme un faux or, le mot a été fait pour dire" (RAMOS, 1962). Les blanchisseuses ne lavent pas les vêtements dans un seul bain. II y a tout un processus pour que, à la fin du lavage, les vêtements soient nettoyés de façon satisfaisante. II en va de même pour le sujet qui écrit. Il est nécessaire d'inventer, de rappeler, de s'approprier, de créer, de reformuler, d'effacer, de choisir, d'élire des sens et de tisser aussi ce qu'est l'écrivain lui-même, c'est-àdire, son identité dans l'écriture, selon Liane Castro (2001). Grâce à cela, le sujet démontrera son autonomie avec la langue / langage dans l'écriture académique.

Source: extrait de 2ème version de recension d'étudiant de la 2ème période .

On peut voir que l'exemple 4 diffère globalement des autres concernant la dimension 
dialogique qu'implique l'auctorialité dans l'écriture académique. Ce point de vue s'appuie, en premier lieu, sur le fait que le discours cité se superpose au discours citant, à la fois par rapport au thème de l'énoncé et par rapport à la disposition spatiale des idées du texte. Cette superposition se manifeste d'abord par l'abondance du recours au discours d'autrui (présence de deux citations et une reformulation dans un seul paragraphe) .

A titre d'exemple, le commentaire qui suit la première citation s'inscrit dans un mouvement paraphrastique proche de la répétition de la perspective énonciative du discours cité. En fait, bien que l'étudiant-auteur ne l'ait pas indiqué, la reformulation colle véritablement au contenu de l'un des textes-source fournis lors de l'activité de rédaction : en comparant le processus d'écriture avec le travail des blanchisseuses, l'étudiant ne produit pas un exemple personnel, mais reprend l'analogie créée par l'auteur cité.

L'adhésion caractérise le positionnement énonciatif de l'étudiant-auteur par rapport au discours cité, si l'on admet que l'absence de marques énonciatives peut être synonyme d'adhésion. Cela dit, précisément en raison de cette absence de marques de prise en charge énonciatives, on ne peut évaluer ni le degré exact de cette adhésion, ni la portée de l'appropriation du discours d'autrui par l'étudiant-auteur. De ce fait, les voix semblent s'orchestrer d'elles-mêmes : "ainsi, dans un seul jet d'un seul souffle, en utilisant des mots uniquement pour exprimer une pensée déjà prête, comme Liane Castro de Araújo dans son texte "Tisser les sens : réécrire et reproduire le texte " (2011), parce que le mot " n'a pas été créé pour orner, pour briller comme un faux or, le mot a été fait pour le dire " (RAMOS, 1962).

Ainsi, malgré les efforts de l'étudiant-auteur pour convoquer différentes sources dans son texte, on n'observe pas ce que Possenti (2001) appelle « un travail de soi dans le discours d'autrui ».

Inventaire des stratégies déployées : tableau récapitulatif

Comme nous l'avons dit, les exemples analysés ont été sélectionnés pour illustrer, à partir des catégories bakhtiniennes retenues, certaines des stratégies que l'étudiant-auteur déploie dans son dialogue avec le discours d'autrui, stratégies qui nous donnent des indices sur la posture d'auctorialité qu'il construit dans son texte. Au-delà de ces exemples, le tableau suivant synthétise l'ensemble des stratégies observées dans l'intégralité de notre corpus. 
Tableau 1 - La dimension dialogique de l'auctorialité

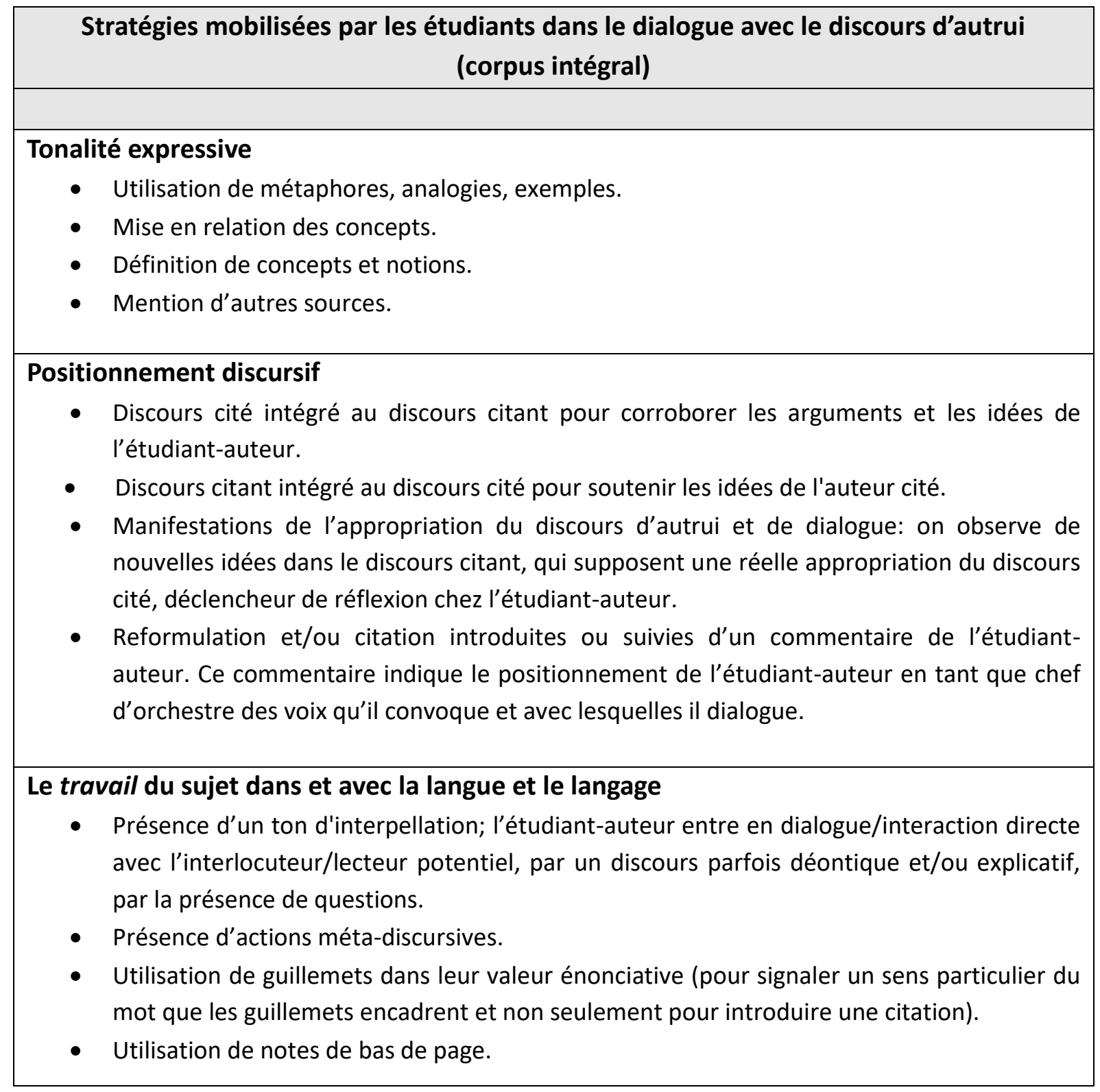

Comme on peut le voir dans cet inventaire, le dialogue avec le discours d'autrui, analysé à partir de sa relation avec les catégories de tonalité expressive, le positionnement discursif et le travail du sujet sur et dans la langue/le langage, se manifeste de manière différente dans les textes analysés, qui utilisent à des degrés divers les stratégies inventoriées pour chacune de ces catégories. En d'autres termes, au plan didactique, adopter cette perspective nécessite de prendre en compte la maîtrise variable, par les étudiants, de I'utilisation de stratégies linguistiques-textuelles, discursives et énonciatives qu'ils mobilisent lorsqu'ils dialoguent ou font dialoguer le réseau de voix convoquées.

$\mathrm{Si}$, comme nous l'avons précisé, ces catégories doivent être considérées de manière 
imbriquée, nous les avons présenté séparément pour des raisons purement méthodologiques. Nos observations nous permettent de constater qu'en ce qui concerne la question du positionnement énonciatif, les difficultés des étudiants se concentrent généralement dans le fait d'adopter un point de vue critique dans le dialogue établi avec le discours d'autrui.

Au delà des quatre exemples détaillés ici, l'analyse globale de nos données montre que les étudiants ne remettent pas en question le discours cité dans le cadre des discussions qu'ils mènent : ils adoptent très majoritairement une position d'adhésion et d'affiliation à ce discours. On voit ainsi, dans la manière dont les étudiants dialoguent avec le discours d'autrui, la prédominance d'une compréhension active plus paraphrasique qu'inédite, du point de vue de la réflexion entreprise dans ce dialogue.

Si l'on se place du point de vue de l'étudiant-auteur, on comprendra aisément cette faible représentativité d'idées nouvelles dans la réflexion : la gestion - toujours complexe de la polyphonie que le néophyte doit assurer en faisant dialoguer des textes d'experts ne favorise pas la prise de distance nécessaire à la naissance d'une voix originale. Sans doute s'agit-il là d'une attente trop forte pour des étudiants encore en formation. Cela étant, de notre point de vue, les activités pédagogiques proposées aux étudiants dans le cadre de formations à l'écriture scientifique doivent favoriser in fine le développement de ce type de compétences.

\section{Premières conclusions}

Les données analysées et discutées montrent que l'analyse des stratégies à l'œuvre dans le dialogue avec le discours d'autrui constitue une porte d'entrée fructueuse pour l'identification d'indices de la manifestation de la position auctoriale dans l'écriture académique.

En d'autres termes, en prenant la dimension du dialogue avec le discours d'autrui comme axe central d'analyse et sa relation avec la construction de la position auctoriale, nous pouvons désormais décrire la manière dont l'auctorialité se construit et se manifeste dans le dialogue au vu des catégories explorées et des stratégies linguistiques-textuelles, discursives et énonciatives mobilisées dans ce dialogue.

Au-delà de ces aspects méthodologiques liés à la recherche, notre analyse nous semble également exploitable dans le cadre didactique : du fait que ces stratégies soient identifiées et illustrées par des exemples concrets émanant de leurs pairs, un travail auprès des étudiants visant une prise de conscience durable de ces stratégies semble pleinement envisageable, afin de les amener à endosser une posture auctoriale plus légitimée. 


\section{Références}

ARAÚJO, Liane Castro de. Tecendo Sentidos: reescrita e produção de texto. Revista da FACED, n. 5, p. 107-115, 2001.

ASSIS, Juliana Alves. Ações do professor e do universitário nas práticas de ensino e de aprendizagem da escrita acadêmica: o papel da avaliação e da reescrita no processo de apropriação do gênero resenha. Eutomia, Recife, v. 13, n. 1, p. 543-561, 2014.

ASSIS, Juliana Alves. Projeto A escrita acadêmica em uma perspectiva dialógica: ser e dizer com e a partir da palavra de outrem. Belo Horizonte, PUC Minas. 2014-2016.

AUTHIER-REVUZ, Jacqueline. Paradas sobre Palavras: a língua em prova na enunciação e na escrita. Educ. Real., Porto Alegre, v. 36, n. 3, p. 651-679, set./dez. 2011.

AUTHIER-REVUZ, Jacqueline. Heterogeneidade(s) enunciativas(s). Cadernos de Estudos Lingüísticos, v. 19, p. 25-42, 1990.

BAKHTIM, Mickail. Estética da criação verbal. Trad. Maria Ermantina Galvão G. Pereira. São Paulo: Martins Fontes. 1997.

BAKHTIN, M. A forma espacial da personagem. In: Estética da criação verbal. Trad. Paulo Bezerra. 6. ed. São Paulo: Editora Martins Fontes, 2011.

$\mathrm{BOCH}$, Françoise. Pratiques d'écriture et de réécriture à l'université: la prise de notes, entre texte source et texte cible. 1998. Thèse de doctorat, Université Stendhal. 1998.

BOCH, Françoise; GROSSMANN, Francis. De l'usage des citations dans le discours théorique: des constats aux propositions didactiques. Lidil, v. 24, p. 91-111, 2001.

COMPAGNON, Antoine. $\mathbf{O}$ trabalho da citação. Trad. Cleonice P. B. Mourão. Belo Horizonte: Editora UFMG. 2007.

GROSSMANN, Francis. Du discours rapporté au discours autorisé, le maniement des noms d'auteur dans les articles en sciences humaines. Estudios de lengua y literaturas franceses, v. 14, p. 9-31, 2003.

GROSSMANN, Francis. Por que e como as coisas mudam? Padronização e variação no campo do discurso científico. In: RINCK, Fanny; BOCH, Françoise; ASSIS, Juliana Alves. (Orgs.) Letramento e formação universitária: formar para a escrita e pela escrita. Campinas, SP: Mercado de Letras, 2015. p. 97- 128.

PAULA, Danytiele Cristina Fernandes de. A questão da identidade na escrita acadêmica, Scripta. v.21, n. 43, p. 86-104, 2017.

POSSENTI, Sírio. O "eu" no discurso do "outro" e a subjetividade mostrada. Alfa, v. 39, p. 4555, 1995.

POSSENTI, Sírio. Indícios de autoria. In: POSSENTI, Sírio. Questões para analistas do discurso São Paulo: Parábola Editorial, 2009. p. 103-118.

PONZIO, Augusto. Alteridade e dialogicidade da palavra. In: Procurando uma palavra outra. São Carlos: Pedro \& João Editores, 2010. p.37-39. 
RAMOS, Graciliano. Linhas Tortas. Rio de Janeiro, Record: 1962. Disponível em: http://graciliano.com.br/site/obra/linhas-tortas-1962. Acesso em: 26 mar. 2018.

REUTER, Yves. De quelques obstacles à l'écriture de recherche. Lidil, v. 17, p. 11-23, 1998.

RINCK, Fanny; BOCH, Françoise; ASSIS, Juliana Alves. Apresentação. In: RINCK, Fanny; BOCH, Françoise; ASSIS, Juliana Alves. (orgs.) Letramento e formação universitária: formar para a escrita e pela escrita. Campinas, SP: Mercado de Letras, 2015. p. 11- 26.

RINCK, Fanny. Former à et par l'écrit de recherche. Quels enjeux, quelles exigences ? Le Français Aujourd'hui, v. 174, p. 79-89, 2011.

Recebido em: 30/03/2019

Aceito em: 25/06/2019 